Haya: The Saudi Journal of Life Sciences

Abbreviated Key Title: Haya Saudi J Life Sci

ISSN 2415-623X (Print) |ISSN 2415-6221 (Online)

Scholars Middle East Publishers, Dubai, United Arab Emirates

Journal homepage: https://saudijournals.com

Original Research Article

\title{
Production and Profitability Study of White Jute Seed at Farmer's Level in Different Areas of Bangladesh
}

\author{
Nasir Uddin ${ }^{1}$, Md. Meftahul Karim ${ }^{1 *}$, Bishwajit Kundu ${ }^{1}$, Md. Babul Hossain ${ }^{1}$ \\ ${ }^{1}$ Bangladesh Jute Research Institute, Dhaka-1207, Bangladesh
}

DOI: $10.36348 /$ sjls.2021.v06i04.001 $\quad$ | Received: 29.01.2021 | Accepted: 16.02.2021 | Published: 25.04 .2021

*Corresponding author: Md. Meftahul Karim

\section{Abstract}

The objective of this study was to investigate the cost and return structures of white jute seed cultivation in Bangladesh. This study was conducted in two locations in three consecutive years 2013-15). The sample included 120 farmers selected using a purposive sampling method, consisting of 60 from each district. Sample size was same for three years. Survey questionnaires were used as the main instrument for data collection. Descriptive statistics and cost and return analysis were used for data analysis. The results indicate that the cost of production (99175 tkha ${ }^{-1}$ ) and output (123502 tkha $^{-1}$ ) was higher in Tangail than Manikganj. Lowest cost of production (80252 tkha $^{-1}$ ) was recorded in 2013 in Manikganj and maximum (102470 tkha ${ }^{-1}$ ) in 2014 in Tangail. Average BCR of white jute seed production of two locations was 1.25. Cost of production varies year to year and place to place due to variation in land rent value, labour wages, land preparation cost etc. This result indicates that profitability of jute seed production is almost same in two zones and by product yield is also important in case of white jute seed production.

Keywords: White jute, Seed yield, Fibre yield, Stick yield, BCR, Profitability.

Copyright (C) 2021 The Author(s): This is an open-access article distributed under the terms of the Creative Commons Attribution 4.0 International License (CC BY-NC 4.0) which permits unrestricted use, distribution, and reproduction in any medium for non-commercial use provided the original author and source are credited.

\section{INTRODUCTION}

Jute (Corchorus spp) is the most important natural fibre crop next to cotton grown in the humid tropical climate mainly under rain fed condition predominantly by marginal and small farmers of IndoBangladesh subcontinent [1]. Jute alone contributes about $1.58 \%$ to GDP without involving any foreign investment [2]. The genus Corchorus belongs to the family Malvaceae, which is composed of approximately 100 species [3]. Of these, two species (Corchorus olitorius L. and Corchorus capsularis L.) are widely cultivated for natural fiber in areas distributed throughout the tropical and sub-tropical regions of the world, particularly in Asia, Africa and Latin America [4]. In Bangladesh Corchorus capsularis L. is known as white jute. About $9 \%$ of total jute cultivable areas are covered by white jute [5].

Quality seed is the prerequisite for increasing the yield and sustainable production of any crop [6]. Seed is an input could increase crop production by 10 $15 \%$ [7]. Jute cultivation area was $10 \%$ of agricultural land area. Jute production was $26 \%$ as of all agricultural crops [23]. Bangladesh requires about 5500-6000 tons jute seeds in every year, of which only $10-15 \%$ is produced and distributed by the BADC [8]. In every year Bangladesh require about 800 metric tons white jute which is totally produced in our country [9]. Farmers show less interest to produce jute seed due to jute seed growing season maximum land were occupied by T. Aman rice and high value vegetables. Encouraging farmers to venture into jute seed production requires relevant information to support their farming decisions. One business tool that provides this information is "cost and return analysis." This crucial tool is used to analyze alternative farming enterprises that can be employed, thereby helping farmers solve practical problems [10]. The cost of production is an important economic indicator that determines farm production performance [11]. This indicator can help farmers with decision making because it considers methods of solving risks associated with agricultural production [12-14]. With the above circumstances, the present study has been undertaken to assess the cost and return of white jute seed production at farm level.

\section{MATERIAL AND METHODS Study area}

The survey was made in 4 upazilla of two districts where white jute seed grown intensively. 


\section{Sample size}

The sample consisted of 120 farmers ( 30 from each location) selected through a purposive sampling technique.

\section{DATA COLLECTION}

Data collection involved personal interviews with the sampled farmers using structured questionnaires. The survey instrument consisted cost and return data associated with jute seed production during the 2013-15 cropping years.

\section{DATA ANALYSIS}

Data analysis consisted of cost and return analysis of white jute seed production, which was divided into 3 parts as follows:

\section{Cost and return analysis}

Cost and return analysis (CRA) is a type of economic analysis that considers costs (both implicit and explicit) incurred by farmers [11,12]. The concepts of Ciaian et al. [11] and Preedasak [15] were followed in this study and are presented as follows:

Cost (i) Total cost (TC) represents the final value of all inputs (cash and noncash) a farm uses in a given period and is the sum of variable and fixed costs.

(ii) Fixed costs (FCs) are independent of the production level.

(iii) Variable costs (VCs) change with the production level.

Both FC and VC can be classified into the explicit costs (cash) and implicit costs (noncash). Explicit costs are the actual expenses incurred, while implicit costs are not associated with the actual expenditure payments.

Return Total revenue (TR) represents the total income that farmers receive from selling agricultural products and by-products per season.

Profit (gross margin): Net profit is the difference between the TR and TC.

In terms of implicit costs, this study adopted the estimation guidelines from Ciaian et al. [11] and Khunthongjan [16]:

(i) Household labor. This represents the cost of hired labor, which was determined using the prevailing market wage rate in the study area, which was varying from 300-350 taka per day.

(ii) Land rent. Farmers' land was assessed on the basis of a local rental rate.

(iii) Depreciation of agricultural machinery. This represents costs accounting for the declining value of farm assets computed using the straight-line method.
Break-even analysis: Break-even analysis is "a technique used to analyze cost information" [17]. It involves the investigation of the level of sales at which a company would make zero profit [18], and it is one of the many techniques that have been developed to aid in management activities such as planning, coordinating, and controlling business operations toward desired success [19]. For this study, break-even analyses of yield and price were adopted from Dillon [20], presented as follows:

Price: Given a known yield and cost, at what market price would the farm "break even" (costs equal income)

Output price $(\mathbf{P i})=\frac{\mathrm{VCi}+\mathrm{FC} i+\pi \mathrm{i}}{\mathrm{Yi}}$

Yield: Given a known price and cost, at what level of production (yield) would the farm break even (costs equal income)

Yield $(\mathrm{Yi})=\frac{\mathrm{VCi}+\mathrm{FCi}+\pi \mathrm{i}}{\mathrm{Pi}}$

Where:

$\mathrm{Pi}$ is the output prices of commodity $\mathrm{i}$;

$\mathrm{Yi}$ is the yield of output $\mathrm{i}$;

$\mathrm{VCi}$ represents the variable costs incurred in the production of commodity $i$;

FCi represents the fixed costs for the production of commodity $i$.

Break-even considerations in just covering costs can be investigated by setting profits $(\pi \mathrm{i})$ equal to zero. The profit, in this case, was not reflected.

\section{Benefit-cost ratio}

Benefit cost ratio is the proportion of net return (benefit) and total cost of production [20]. The $\mathrm{B}-\mathrm{C}$ ratio was calculated by dividing the gross margin by the total variable production cost.

\section{RESULT AND DISCUSSION Cost of white jute seed production}

Cost items for jute seed production were divided into variable and fixed costs (Table 1 and 2). The average total cost of jute seed production in Manikganj was $86024 \mathrm{tkha}^{-1}$, where in total variable and fixed cost accounted for 80.2 and $19.8 \%$ of the overall cost, respectively. Labor cost associated $66 \%$ of total variable cost and land rent cover $19.4 \%$ of total cost. Noncash labor cost was also estimated from the prevailing labor wage as a shadow price for family labor. Findings showed that land rent responsible for maximum amount of fixed cost and $19.4 \%$ of total cost. Pesticide, seed, irrigation and depreciation cost account for negligible amount considering the total cost. Fertilizer and land preparation were two important facts those cover $5.6 \%$ and $7.2 \%$ of total cost respectively. 
For white Jute seed production irrigation cost is very low in Manikganj it is less than $1 \%$. Most of the jute seed production farmers in the Manikganj used their own land in farming and land rent was low because for white jute seed production farmers mainly use their marginal land. Result showed that depreciation cost was very marginal because farmer used very small amount of machinery for white jute seed production.

The average output of the jute seed was 608 $\mathrm{kg} / \mathrm{ha}$, with a prevailing market price of $100 \mathrm{tkkg}^{-1}$, allowing farmers to have a revenue of $60800 \mathrm{tkha}^{-1}$. In white jute seed production fibre and stick are two important by-products. By-product yield in the study presented as seed equivalent yield. Total yield containing seed equivalent yield of fibre and stick was $1008 \mathrm{kgha}^{-1}$, allowing farmers to have return of 100800 $\mathrm{tkha}^{-1}$. Average benefit cost ratio for white jute production was found 1.17 in Manikganj. BCR was below the expectation level because of low seed yield and value. Break even analysis indicates that average price of white seed should be minimum $85.34 \mathrm{tkkg}^{-1}$ in Manikganj.

In Tangail the average total cost of white jute seed production was recorded $99175 \mathrm{tkha}^{-1}$. Labor cost was the major cost for white jute seed production that associated $57.85 \%$ of total variable cost and land rent cover $24.91 \%$ of total cost. Noncash labor cost was also estimated from the prevailing labor wage as a shadow price for family labor. Among fixed cost land rent was account for major portion whereas depreciation covers only very little amount because farmer use only a few machinery for white jute seed production. Pesticide, seed, and irrigation cost account for negligible amount considering the total cost. Some farmers used their own seed that consider as non-cash cost. Most of the jute seed cultivars in Tangail utilized their own land in farming that value was considered as non-cash cost and land rent was low because for white jute seed production farmers mainly make use of their marginal land.

This study exposed the seed yield of white jute in three consecutive years in Tangail fluctuated from 620 to $678 \mathrm{kgha}^{-1}$ and in average it was $647 \mathrm{kgha}^{-1}$ with a prevailing average market price of $98.33 \mathrm{tkkg}^{-1}$, allowing farmers to have a revenue of 63619 tkha $^{-1}$. Considering the seed equivalent yield of fibre and stick total seed equivalent yield of white jute was $1256 \mathrm{kgha}^{-}$ ${ }^{1}$ in Tangail. Average benefit cost ratio for white jute production was 1.25 . Break even analysis indicates that average price white seed should be minimum $78.4 \mathrm{tk} / \mathrm{kg}$ in Tangail.
In average white jute seed production of two locations expenses for labor and land rent were the highest (Table 3), accounting for 61.49 and $22.29 \%$ of the total production cost. Similar labor cost was also found by Islam and Uddin 2019. In Tangail land rent was higher compare to the Manikganj because Tangail is more agriculture dependent area than Manikganj and land productivity is higher. Fertilizer cost of white jute seed production is very low because jute is an ecofriendly crop [21] that needs very low dose of fertilizer additionally it's add organic matter to the soil. Land preparation cost was similar in both areas on an average it was $6.67 \%$ of the total cost. Pest management cost was very low $(0.68 \%)$ percent of total cost because in direct seeding method pest infestation very low [22]. Total variable cost cover $77.27 \%$ cost that is almost about 3.5 times higher than total fixed cost. Average total fixed cost of two locations was $92599 \mathrm{tkha}^{-1}$. Total cash cost cover $59.27 \%$ of total cost of white jute seed production and in non-cash cost family labor cover $60.87 \%$ of total non-cash cost.

Seed price variation in two locations was very marginal, average seed price of two locations was 99.17 $\mathrm{tkkg}^{-1}$. Seed production was higher in Tangail over Manikganj and average seed production of two locations was $627 \mathrm{kgha}^{-1}$. This yield is low compare to the yield obtain by karim et al. [9] and Islam et al. [22]. Average seed equivalent yield of jute fibre and jute stick was $505 \mathrm{kgha}^{-1}$. Normally value of white jute fibre varies from 42 to $55 \mathrm{tkkg}^{-1}$ but in case of seed production fibre is the by-product. That fibre is low quality fibre because crop remains in the field more than 120 day as result cuttings percentage increase for that reason its value is low. Average jute fibre price was $27.12 \mathrm{tkkg}^{-1}$. Considering the jute fibre and stick average gross return of two locations was 112260 tkha 1 . Gross return and gross margin was higher in Tnagail over Manikganj due to high jute seed and byproduct yield. This study found average breakeven price of white jute seed was $82 \mathrm{tkkg}^{-1}$ seed. The results regarding the breakeven price reflect the minimum price required to recover the unit cost of production. Result showed that BCR was higher in Tangail and average BCR of white jute seed production was 1.21. Islam and uddin [22] reported 1.25 BCR for white jute seed production. BCR for white jute seed production was low because farmer did not use update production technology for that cause seed yield was low moreover quality of was not up to the mark for that value of seed was low. Labor wages was higher in these locations that increased the cost of production. 
Nasir Uddin et al., Haya Saudi J Life Sci, Apr, 2021; 6(4): 56-62

Table-1: Cost and return of white jute seed production in Manikganj (2013-2015)

\begin{tabular}{|c|c|c|c|c|c|c|c|c|c|c|c|c|c|}
\hline \multirow[t]{2}{*}{ Items } & \multicolumn{3}{|l|}{2013} & \multicolumn{3}{|l|}{2014} & \multicolumn{3}{|l|}{2015} & \multicolumn{4}{|c|}{ Average } \\
\hline & Cash & $\begin{array}{l}\text { Non } \\
\text { Cash }\end{array}$ & Total & Cash & $\begin{array}{l}\text { Non } \\
\text { cash }\end{array}$ & Total & Cash & $\begin{array}{l}\text { Non } \\
\text { Cash }\end{array}$ & Total & Cash & $\begin{array}{l}\text { Non } \\
\text { Cash }\end{array}$ & Total & $\begin{array}{l}\text { Percen } \\
\text { tage }\end{array}$ \\
\hline \multicolumn{14}{|l|}{$\begin{array}{l}\text { 1.Variable cost } \\
(\mathrm{VCs})^{*}\end{array}$} \\
\hline Labor & $\begin{array}{l}3375 \\
0\end{array}$ & $\begin{array}{l}2250 \\
0\end{array}$ & $\begin{array}{l}5625 \\
0\end{array}$ & 35750 & $\begin{array}{l}2125 \\
0\end{array}$ & $\begin{array}{l}5700 \\
0\end{array}$ & 34960 & $\begin{array}{l}2215 \\
4\end{array}$ & $\begin{array}{l}5711 \\
4\end{array}$ & 34820 & $\begin{array}{l}2196 \\
8\end{array}$ & $\begin{array}{l}5678 \\
8\end{array}$ & 66 \\
\hline Seed & 600 & 200 & 800 & 500 & 250 & 750 & 300 & 465 & 765 & 466 & 305 & 771 & 0.90 \\
\hline $\begin{array}{l}\text { Land } \\
\text { preparation }\end{array}$ & 5500 & 600 & 6100 & 5600 & 650 & 6250 & 5750 & 560 & 6310 & 5616 & 603 & 6219 & 7.2 \\
\hline Fertilizer & 4900 & 540 & 5440 & 3850 & 507 & 4357 & 4234 & 351 & 4585 & 4328 & 466 & 4794 & 5.60 \\
\hline Pesticide & 62 & & 62 & 500 & & 500 & & & & 187 & & 187 & 0.22 \\
\hline Irrigation & & & & 750 & & 750 & & & & 250 & & 250 & 0.30 \\
\hline $\begin{array}{l}\text { Total } \\
\text { Variable cost } \\
\text { (TVC) }\end{array}$ & $\begin{array}{l}4481 \\
2\end{array}$ & $\begin{array}{l}2384 \\
0\end{array}$ & $\begin{array}{l}6865 \\
2\end{array}$ & 46950 & $\begin{array}{l}2265 \\
7\end{array}$ & $\begin{array}{l}6960 \\
7\end{array}$ & 45244 & $\begin{array}{l}2353 \\
0\end{array}$ & $\begin{array}{l}6877 \\
4\end{array}$ & 45668 & $\begin{array}{l}2334 \\
2\end{array}$ & $\begin{array}{l}6901 \\
1\end{array}$ & 80.2 \\
\hline \multicolumn{14}{|l|}{$\begin{array}{l}\text { 2.Fixed Cost } \\
(\mathrm{FCs})^{*}\end{array}$} \\
\hline Land rent & 3000 & 8250 & $\begin{array}{l}1125 \\
0\end{array}$ & 4600 & $\begin{array}{l}1415 \\
0\end{array}$ & $\begin{array}{l}1875 \\
0\end{array}$ & 5200 & $\begin{array}{l}1480 \\
0\end{array}$ & $\begin{array}{l}2000 \\
0 \\
\end{array}$ & 4266 & $\begin{array}{l}1240 \\
0\end{array}$ & $\begin{array}{l}1666 \\
6 \\
\end{array}$ & 19.4 \\
\hline $\begin{array}{l}\text { Depreciation } \\
\text { cost }\end{array}$ & & 350 & 350 & & 370 & 370 & & 320 & 320 & & 346 & 346 & 0.40 \\
\hline $\begin{array}{l}\text { Total Fixed } \\
\text { Cost (TFC) }\end{array}$ & 3000 & 8600 & $\begin{array}{l}1160 \\
0\end{array}$ & 4600 & $\begin{array}{l}1452 \\
0\end{array}$ & $\begin{array}{l}1912 \\
0\end{array}$ & 5200 & $\begin{array}{l}1512 \\
0\end{array}$ & $\begin{array}{l}2032 \\
0\end{array}$ & 4266 & $\begin{array}{l}1274 \\
6 \\
\end{array}$ & $\begin{array}{l}1701 \\
2\end{array}$ & 19.8 \\
\hline $\begin{array}{l}\text { Total cost } \\
(\mathrm{TVC}+\mathrm{TFC})^{*}\end{array}$ & $\begin{array}{l}4781 \\
2 \\
\end{array}$ & $\begin{array}{l}3244 \\
0 \\
\end{array}$ & $\begin{array}{l}8025 \\
2 \\
\end{array}$ & 51550 & $\begin{array}{l}3717 \\
7 \\
\end{array}$ & $\begin{array}{l}8872 \\
7 \\
\end{array}$ & 50444 & $\begin{array}{l}3865 \\
0 \\
\end{array}$ & $\begin{array}{l}8909 \\
4 \\
\end{array}$ & 49935 & $\begin{array}{l}3608 \\
9 \\
\end{array}$ & $\begin{array}{l}8602 \\
4 \\
\end{array}$ & \\
\hline $\begin{array}{l}\text { Price of seed } \\
\text { (p) }(\mathrm{Tk} / \mathrm{kg})\end{array}$ & 85 & & & 105 & & & 110 & & & 100 & & & \\
\hline $\begin{array}{l}\text { Price of Fibre } \\
(\mathrm{Tk} / \mathrm{kg})\end{array}$ & 20 & & & 30 & & & 30 & & & 26.67 & & & \\
\hline $\begin{array}{l}\text { Price of stick } \\
(\mathrm{Tk} / \mathrm{kg})\end{array}$ & 7 & & & 5 & & & 6 & & & 6 & & & \\
\hline \multicolumn{14}{|l|}{$\begin{array}{l}\text { Production } \\
(\mathrm{kg} / \mathrm{ha})\end{array}$} \\
\hline Seed $(\mathrm{s})$ & 625 & & & 610 & & & 590 & & & 608 & & & \\
\hline Fibre & 1005 & & & 1100 & & & 1070 & & & 1058 & & & \\
\hline Stick & 1900 & & & 2020 & & & 2010 & & & 1976 & & & \\
\hline $\begin{array}{l}\text { Seed } \\
\text { equivalent } \\
\text { yield of fibre } \\
\text { (f) } \\
\end{array}$ & 236 & & & 314 & & & 291 & & & 282 & & & \\
\hline $\begin{array}{l}\text { Seed } \\
\text { equivalent } \\
\text { yield of stick } \\
\text { (v) } \\
\end{array}$ & 156 & & & 96 & & & 109 & & & 118 & & & \\
\hline $\begin{array}{l}\text { Total Seed } \\
\text { equivalent } \\
\text { yield }(y)(s+\mathbf{f} \\
+\mathrm{v})\end{array}$ & 1017 & & & 1020 & & & 990 & & & 1008 & & & \\
\hline $\begin{array}{l}\text { Gross Return } \\
\left(y^{*} p\right)(T k / h a)\end{array}$ & $\begin{array}{l}8644 \\
5 \\
\end{array}$ & & & $\begin{array}{l}10710 \\
0\end{array}$ & & & $\begin{array}{l}10890 \\
0\end{array}$ & & & $\begin{array}{l}10080 \\
0\end{array}$ & & & \\
\hline $\begin{array}{l}\text { Gross margin } \\
\text { (profit) }\end{array}$ & 6193 & & & 18373 & & & 19806 & & & 14776 & & & \\
\hline $\begin{array}{l}\text { Break-even } \\
\text { quantity } \\
\text { (kg/seed) }\end{array}$ & 944 & & & 845 & & & 810 & & & 860 & & & \\
\hline $\begin{array}{l}\text { Break-even } \\
\text { price }(\mathrm{tk} / \mathrm{kg} \\
\text { seed) }\end{array}$ & 78.91 & & & 87 & & & 90 & & & 85.34 & & & \\
\hline $\begin{array}{l}\text { Benefit-cost } \\
\text { ratio (B:C) }\end{array}$ & 1.08 & & & 1.21 & & & 1.22 & & & 1.17 & & & \\
\hline
\end{tabular}


Nasir Uddin et al., Haya Saudi J Life Sci, Apr, 2021; 6(4): 56-62

Table 2: Cost and return of white jute seed production in Tangail (2013-2015)

\begin{tabular}{|c|c|c|c|c|c|c|c|c|c|c|c|c|c|}
\hline \multirow[t]{2}{*}{ Items } & \multicolumn{3}{|l|}{2013} & \multicolumn{3}{|l|}{2014} & \multicolumn{3}{|l|}{2015} & \multicolumn{3}{|c|}{ Average } & \multirow[b]{2}{*}{$\begin{array}{l}\text { Percent } \\
\text { age }\end{array}$} \\
\hline & Cash & $\begin{array}{l}\text { Non } \\
\text { Cash } \\
\end{array}$ & Total & Cash & $\begin{array}{l}\text { Non } \\
\text { cash }\end{array}$ & Total & Cash & $\begin{array}{l}\text { Non } \\
\text { Cash } \\
\end{array}$ & Total & Cash & $\begin{array}{l}\text { Non } \\
\text { Cash } \\
\end{array}$ & Total & \\
\hline \multicolumn{14}{|l|}{$\begin{array}{l}\text { 1. Variable } \\
\text { cost }(\mathrm{VCs})^{*}\end{array}$} \\
\hline Labor & 35100 & 24750 & 59850 & 34154 & 22155 & 56309 & 33960 & $\begin{array}{l}2199 \\
5\end{array}$ & 55955 & 34404 & 22967 & 57371 & 57.85 \\
\hline Seed & 622.5 & 200 & 822 & 407 & 471 & 878 & 446.2 & 412 & 858 & 492 & 361 & 853 & 0.86 \\
\hline $\begin{array}{l}\text { Land } \\
\text { preparation }\end{array}$ & 5550 & 510 & 6060 & 5864 & 310 & 6174 & 5960 & 297 & 6257 & 5791 & 372 & 6163 & 6.21 \\
\hline Fertilizer & 8050 & & 8050 & 8590 & & 8590 & 7341 & & 7341 & 7993 & & 7993 & 8.06 \\
\hline Pesticide & 1018 & & 1018 & 1290 & & 1290 & 900 & & 900 & 1069 & & 1069 & 1.08 \\
\hline Irrigation & 336 & & 336 & - & - & - & 1290 & & 1290 & 542 & & 542 & 0.55 \\
\hline $\begin{array}{l}\text { Total } \\
\text { Variable } \\
\text { cost (TVC) }\end{array}$ & 50679 & 25460 & 76139 & 50306 & 22937 & 73243 & 49897 & $\begin{array}{l}2270 \\
4\end{array}$ & 72601 & 50294 & 23700 & 73994 & 74.61 \\
\hline \multicolumn{14}{|l|}{$\begin{array}{l}\text { 2.Fixed Cost } \\
(\mathrm{FCs})^{*}\end{array}$} \\
\hline Land rent & 6009 & 12609 & 18618 & 12567 & 16183 & 28750 & 10029 & $\begin{array}{l}1673 \\
0\end{array}$ & 26759 & 9535 & 15174 & 24709 & 24.91 \\
\hline $\begin{array}{l}\text { Depreciation } \\
\text { cost }\end{array}$ & & 564 & 564 & & 475 & 475 & & 372 & 372 & & 471 & 471 & 0.48 \\
\hline $\begin{array}{l}\text { Total Fixed } \\
\text { Cost (TFC) }\end{array}$ & 6009 & 13173 & 19182 & 12567 & 16659 & 29226 & 10029 & $\begin{array}{l}1710 \\
3 \\
\end{array}$ & 27132 & 9535 & 15645 & 25180 & 25.39 \\
\hline $\begin{array}{l}\text { Total cost } \\
(\mathrm{TVC}+ \\
\text { TFC)* }\end{array}$ & 56688 & 38634 & 95322 & 62874 & 39596 & 102470 & 59927 & $\begin{array}{l}3980 \\
8\end{array}$ & 99735 & 59829 & 39346 & 99175 & \\
\hline $\begin{array}{l}\text { Price of seed } \\
\text { (p) }(\mathrm{Tk} / \mathrm{kg})\end{array}$ & 80 & & & 105 & & & 110 & & & 98.33 & & & \\
\hline $\begin{array}{l}\text { Price of } \\
\text { Fibre } \\
(\mathrm{Tk} / \mathrm{kg})\end{array}$ & 20 & & & 31.67 & & & 31 & & & 27.56 & & & \\
\hline $\begin{array}{l}\text { Price of stick } \\
(\mathrm{Tk} / \mathrm{kg})\end{array}$ & 7 & & & 4 & & & 5 & & & 5.33 & & & \\
\hline \multicolumn{14}{|l|}{$\begin{array}{l}\text { Production } \\
\text { (kg/ha) }\end{array}$} \\
\hline Seed (s) & 643 & & & 678 & & & 620 & & & 647 & & & \\
\hline Fibre & 1530 & & & 1573 & & & 1600 & & & 1567 & & & \\
\hline Stick & 3323 & & & 2878 & & & 3250 & & & 3150 & & & \\
\hline $\begin{array}{l}\text { Seed } \\
\text { equivalent } \\
\text { yield of fibre } \\
\text { (f) }\end{array}$ & 382 & & & 474 & & & 450 & & & 439 & & & \\
\hline $\begin{array}{l}\text { Seed } \\
\text { equivalent } \\
\text { yield of stick } \\
\text { (v) }\end{array}$ & 290 & & & 109 & & & 147 & & & 170 & & & \\
\hline $\begin{array}{l}\text { Total Seed } \\
\text { equivalent } \\
\text { yield }(y)(s+ \\
\text { f }+v)\end{array}$ & 1315 & & & 1261 & & & 1217 & & & 1256 & & & \\
\hline $\begin{array}{l}\text { Gross } \\
\text { Return } \\
\left(\mathbf{y}^{*} \mathbf{p}\right) \\
(\mathrm{Tk} / \mathbf{h a}) \\
\end{array}$ & 105200 & & & 132405 & & & 133870 & & & $\begin{array}{l}12350 \\
2\end{array}$ & & & \\
\hline $\begin{array}{l}\text { Gross } \\
\text { margin } \\
\text { (profit) }\end{array}$ & 9878 & & & 29935 & & & 34315 & & & 24327 & & & \\
\hline $\begin{array}{l}\text { Break-even } \\
\text { quantity } \\
(\mathrm{kg} / \mathrm{ha})\end{array}$ & 1191 & & & 975 & & & 906 & & & 1008 & & & \\
\hline $\begin{array}{l}\text { Break-even } \\
\text { price }(\mathrm{tk} / \mathrm{kg} \\
\text { fibre) }\end{array}$ & 72.5 & & & 81.2 & & & 82 & & & 78.4 & & & \\
\hline $\begin{array}{l}\text { Benefit-cost } \\
\text { ratio }(\mathrm{B}: \mathrm{C})\end{array}$ & 1.10 & & & 1.29 & & & 1.34 & & & 1.25 & & & \\
\hline
\end{tabular}


Nasir Uddin et al., Haya Saudi J Life Sci, Apr, 2021; 6(4): 56-62

Table-3: Average cost and return of white jute seed production in Manikganj and Tangail

\begin{tabular}{|c|c|c|c|c|c|c|c|c|c|c|}
\hline \multirow[b]{2}{*}{ Items } & \multicolumn{3}{|c|}{ Manikganj } & \multicolumn{3}{|l|}{ Tangail } & \multicolumn{4}{|l|}{ Average } \\
\hline & Cash & $\begin{array}{l}\text { Non } \\
\text { Cash }\end{array}$ & Total & Cash & $\begin{array}{l}\text { Non } \\
\text { cash }\end{array}$ & Total & Cash & $\begin{array}{l}\text { Non } \\
\text { Cash }\end{array}$ & Total & Percentage \\
\hline \multicolumn{11}{|l|}{ 1.Variable cost $(\mathrm{VCs})^{*}$} \\
\hline Labor & 34820 & 21968 & 56788 & 34404 & 22967 & 57371 & 34612 & 22467 & 57079 & 61.49 \\
\hline Seed & 466 & 305 & 771 & 492 & 361 & 853 & 479 & 333 & 812 & 0.87 \\
\hline Land preparation & 5616 & 603 & 6219 & 5791 & 372 & 6163 & 5703 & 487 & 6190 & 6.67 \\
\hline Fertilizer & 4328 & 466 & 4794 & 7993 & & 7993 & 6160 & 466 & 6626 & 7.13 \\
\hline Pesticide & 187 & & 187 & 1069 & & 1069 & 628 & & 628 & 0.68 \\
\hline Irrigation & 250 & & 250 & 542 & & 542 & 396 & & 396 & 0.43 \\
\hline Total Variable cost (TVC) & 45668 & 23342 & 69011 & 50294 & 23700 & 73994 & 47978 & 23753 & 71731 & 77.27 \\
\hline \multicolumn{11}{|l|}{ 2.Fixed Cost $(\mathrm{FCs}) *$} \\
\hline Land rent & 4266 & 12400 & 16666 & 9535 & 15174 & 24709 & 6900 & 13787 & 20687 & 22.29 \\
\hline Depreciation cost & & 346 & 346 & & 471 & 471 & & 408 & 408 & 0.44 \\
\hline Total Fixed Cost (TFC) & 4266 & 12746 & 17012 & 9535 & 15645 & 25180 & 6900 & 14195 & 21095 & 22.73 \\
\hline Total cost $(\mathrm{TVC}+\mathrm{TFC})^{*}$ & 49935 & 36089 & 86024 & 59829 & 39346 & 99175 & 54878 & 37948 & 92826 & \\
\hline Price of seed $(\mathrm{p})(\mathrm{Tk} / \mathrm{kg})$ & 100 & & & 98.33 & & & 99.17 & & & \\
\hline Price of Fibre $(\mathrm{Tk} / \mathrm{kg})$ & 26.67 & & & 27.56 & & & 27.12 & & & \\
\hline Price of stick $(\mathrm{Tk} / \mathrm{kg})$ & 6 & & & 5.33 & & & 5.67 & & & \\
\hline \multicolumn{11}{|l|}{ Production (kg/ha) } \\
\hline Seed (s) & 608 & & & 647 & & & 627 & & & \\
\hline Fibre & 1058 & & & 1567 & & & 1312 & & & \\
\hline Stick & 1976 & & & 3150 & & & 2563 & & & \\
\hline $\begin{array}{l}\text { Seed equivalent yield of fibre } \\
\text { (f) }\end{array}$ & 282 & & & 439 & & & 359 & & & \\
\hline $\begin{array}{l}\text { Seed equivalent yield of stick } \\
\text { (v) }\end{array}$ & 118 & & & 170 & & & 146 & & & \\
\hline $\begin{array}{l}\text { Total Seed equivalent yield } \\
(y)(s+f+v)\end{array}$ & 1008 & & & 1256 & & & 1132 & & & \\
\hline Gross Return $\left(y^{*} p\right)(T k / h a)$ & 100800 & & & 123502 & & & 112260 & & & \\
\hline Gross margin (profit) & 14776 & & & 24327 & & & 19661 & & & \\
\hline Break-even quantity (kg/ha) & 860 & & & 1008 & & & 933 & & & \\
\hline $\begin{array}{l}\text { Break-even price }(\mathrm{tk} / \mathrm{kg} \\
\text { fibre) }\end{array}$ & 85.34 & & & 78.4 & & & 82 & & & \\
\hline Benefit-cost ratio (B:C) & 1.17 & & & 1.25 & & & 1.21 & & & \\
\hline
\end{tabular}

\section{CONCLUSION}

This study investigated the cost and return structure of white jute seed production systems in two location of Bangladesh. Higher variable costs were incurred in Tangail over Manikganj because more variable inputs were used during production. Labor costs represent the largest percentage share of variable costs in both locations. This study revealed that farmers received marginal amount of profit in white jute seed production. Seed production should be increased by using modern technology and use of machinery should increase to reduce the cost of production.

\section{ACKNOWLEDGMENTS}

The authors wish to give thanks to jute seed producer in Manikganj and Tangail for their cooperation in actively participating in the conduct of the research project.

\section{REFERENCES}

1. Das, M., Poddar, P., Haque, S., Pati, S., Poddar, R. and Kundu, C. K. (2014). Yield and economics of white jute as influenced by different dates of sowing, spacing and topping schedule in Terai region of West Bengal. In. J. of Farm Sci, 4(4): 51 $-58$
2. BBS. (2018). Statistical Year book of Bangladesh. Bangladesh Bureau of Statistics. Ministry of Planning and Statistics Division, Govt. of People's Republic of Bangladesh, Dhaka. 170. Website:

3. Saunders, M. (2001). Recovery plan for the endangered native jute species, Corchorus cunninghamii F. Muell in Queensland (2001-2006). Natural Heritage Trust, Australia, 1-29.

4. Hossain, M. B., Haque, S., \& Khan, H. (2002). DNA Fingerprinting of jute germplasm by RAPD. J. Biochem. Mol. Biol. 35:414-419. https://doi.org/10.5483/BMBRep.2002.35.4.414

5. Saha, C. K. (2011). Jute Seed Management in Bangladesh. Proceedings, International Seminar on Strengthening of Collaboration for Jute, Kenaf and Allied Fibres Research and Development, 2011. International Jute Study Group (IJSG), Dhaka, Bangladesh.

6. Abebe, G., \& Alemu, A. (2017). Role of improved seeds towards improving livelihood and food security at Ethiopia, int. j. of res. Granthaalayah, $5(2): 338-356$.

Https://doi.org/10.5281/zenodo.376076

7. Mondal, M. M. (2005). Challenges and Opportunities of sustainable crop production in Bangladesh. Eighth Biennial Agronomy 
Convention. Bangladesh Society of Agronomy, 3236

8. Ali, S., Haque, M., Siddique, A. B., Mollah, A. F., \& Islam, M. N. (2003). Phenology growth and seed yield of tossa jute (Corchorus olitorius L.) in late sown technology. Bangladesh J. of Agri. 27 \& 28: 91-97.

9. Karim, M. M., Rahman, M. L., Ferdush, J., Tareq, M. Z., Miah, M. M., Sultan, M. T. and Himel, R. M. (2020). Yield, quality and cost of jute (Corchorus sp.) seed production as influenced by herbicide application time. Int. J. Advan. Geosci, 8(2), 153-159.

10. Lessley, B. V., Johnson, D. M., \& Hanson, J. C. (1991). Using the partial budget to analyze farm change. University System of Maryland, Maryland

11. Ciaian, P., Paloma, S. G., \& Delince, J. (2013). Literature review on cost of production methodologies: draft version. Food and Agriculture Organization of the United Nations. FAO Headquarters, Rome

12. Netayarak, P. (2007). Agricultural economics. Thammasat University Press, Bangkok

13. Puttikorn, B., Kao-ian, S., \& Sirijinda, A. (2006). Agricultural production economics. Kasetsart University Press, Bangkok

14. Thongpan, S. (2013). The adaptation strategies of farmers in frequently flooded areas in Chumsang District, Nakhon Sawan. Parichart J, 26(3):78-89

15. Preedasak, P. (2004). Principles of microeconomics, 4th edn. Thammasat University Press, Bangkok

16. Khunthongjan, S. (2016). Pattern of income and spending, household rice farmers in Ubon Ratchathani province, Thailand. Silpakorn Univ. J. Soc. Sci. Hum. Arts, 16(1):163-188. https://doi.org/10.14456/sujsha.2016.2
17. Brinckerhoff, P. C., (2009). Mission-based management: leading your not-for-profit in the $21 \mathrm{st}$ century (3rd ed.). Hoboken, NJ: John Wiley \& Sons

18. Tsorakidis, N., Papadoulos, S., Zerres, M., \& Zerres, C. (2008). Breakeven analysis. Business Sumup. https://bit.ly/33nrZPN. Accessed 12 August 2019

19. Dubas, K., Hershey, L., Nijhawan, I., \& Mehta, R. (2011). Breakeven and profitability analyses in marketing management using $\mathrm{R}$ software. Innov. Mark, 7(3):51-61.

20. Dillon, C. (1992). Microeconomic effects of reduced yield variability cultivars of soybeans and wheat. South J. Agric. Econ. 24(1):121-133. https://doi.org/10.1017/S0081305200026042

21. Mir, R. R., Rustgi, S., Sharma, S., Singh, R., Goyal, A., Kumar, J., Gaur, A., Tyagi, A. K., Khan, H., Sinha, M. K., Balyan, H. S., \& Gupta, P. K. (2008). A preliminary genetic analysis of fiber traits and the use of new genomic SSRs for genetic diversity in jute. Euphytica. 161:413-42. https://doi.org/10.1007/s10681-007-9597-x

22. Islam, M. M., \& Uddin, M. N. (2019). Research and Development Advances of Jute Seed in Bangladesh: A.

23. IJSG. (2012). World Jute \& Kenaf Statistics: at a Glance, Jute, Kenaf Other Bast and Hard Fibres: Farm to Fashion, Published by: International Jute Study Group (IJSG) IJSG Secretariat, 145, Monipuripara, Near Farmgate, Tejgoan, Dhaka1215, Bangladesh, Web: www.jute.org

24. Dillon, J. L., \& Hardaker, J. B. (1993). Farm management research for small farm development. Food and Agriculture Organization of United Nations, Rome, Italy. 